\title{
Quantitative STEM-EELS Imaging of Ferritin Distributions in Cultured Erythroblasts Undergoing Erythropoiesis
}

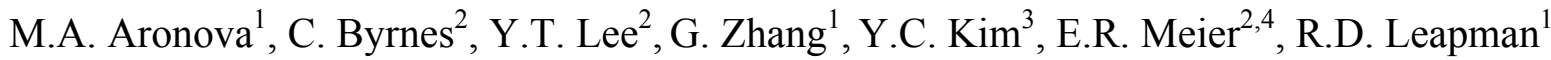 \\ ${ }^{1}$ National Institute of Biomedical Imaging and Bioengineering, NIH, Bethesda, MD \\ ${ }^{2}$ National Institute of Diabetes and Digestive and Kidney Diseases, NIH, Bethesda MD \\ ${ }^{3}$ Center for Materials Physics and Technology, NRL, Washington, DC \\ ${ }^{4}$ Children's National Health System, Washington, DC
}

The process of erythropoiesis whereby erythrocytes, i.e., red blood cells (RBCs), are produced in the body involves continuous regeneration of erythroblasts from hematopoietic stem cells, and their subsequent differentiation into enucleated RBCs. There has been much recent interest in ex vivo erythropoiesis as an approach to tissue engineering of blood, which is one of the reasons why we have explored the structural changes that occur in cultured human erythroblasts during their highly orchestrated differentiation [1]. One important aspect of erythropoiesis is the enormous uptake of iron that is needed to fill the RBCs with hemoglobin, which in mammals constitutes $96 \%$ of red blood cells' dry content. A molecule of hemoglobin contains four heme groups, each of which consists of an iron atom coordinated to a porphyrin ring. There are approximately $2.8 \times 10^{8}$ hemoglobin molecules in each RBC, or more than $10^{9} \mathrm{Fe}$ atoms in a volume of $\sim 10^{11} \mathrm{~nm}^{3}$. Extracellular iron must be transported by transferrin receptors across the plasma membrane, and then to mitochondria for heme synthesis, before being released back to the cytoplasm where it combines with globins. During erythropoiesis, the differentiating erythroblasts must produce enormous quantities of heme and hemoglobin, while also maintaining sufficient cellular machinery to carry out protein synthesis. It is therefore not surprising that the iron storage protein, ferritin, has a role in erythropoiesis [2]; each ferritin molecule can store up to $\sim 4000 \mathrm{Fe}$ atoms in its 8 -nm diameter mineralized ferrihydrite-like core.

Here, we have mapped and quantified the distribution of ferritin in differentiating erythroblasts. To achieve this, we have performed electron energy loss spectroscopic (EELS) imaging in a scanning transmission electron microscope (STEM) [3,4]. Spectrum-images were acquired at a beam energy of $300 \mathrm{keV}$ using a Tecnai TF30 TEM (Thermo Fisher Scientific), equipped with a Quantum GIF (Gatan Inc.) at energy losses in the region of $450 \mathrm{eV}-1400 \mathrm{eV}$, which includes the $\mathrm{Fe} \mathrm{L}_{2,3}$ edge at $710 \mathrm{eV}$. Initially, we acquired STEM images with the high-angle annular dark field (HAADF) detector (Figs. $1 \mathrm{~A}, 1 \mathrm{~B}, 1 \mathrm{C})$, which revealed bright punctate intensities in vesicles located in proximity to mitochondria. Then, spectrum-images were acquired using the dual-EELS mode (acquisition of concurrent core-edge and zero-loss/low-loss spectra) to enable precise calibration of the energy losses throughout the spectrum-image, as well as determination of the number of iron atoms per pixel in elemental maps.

We examined ex vivo erythroid cultures of primary human CD34+ cells, for accumulation of iron during 4 developmental stages: $\mathrm{t} 1=0$ days (hematopoietic progenitor cells) $[\mathrm{CD} 34(+)]$, $\mathrm{t} 2=7$ days (erythroblast progenitor cells) [CD36(+), CD235(-)], t3 $=14$ days (erythropoietin-dependent erythroblasts) $[\mathrm{CD} 36(+), \mathrm{CD} 235(+)]$, and $\mathrm{t} 4=21$ days (nucleated erythroblasts and enucleated erythrocytes). The hormone, erythropoietin, was added to the cultures at $\mathrm{t} 2$. Quantitative iron distributions were computed from the integrated $\mathrm{Fe} \mathrm{L}_{2,3}$-edge signal, from the number of electrons in 
the incident probe (i.e., zero-loss/low-loss intensity), and from the $\mathrm{Fe}_{2,3}$ core-loss cross section (Fig 1D). The iron maps confirmed that the particles in the vesicles were ferritin cores since they each contained $\sim 2,000$ to 3,000 Fe atoms. The number of ferritin cores was least for $\mathrm{t} 1$ cells, and highest for $t 4$ cells. Our results indicate that, in cultured differentiating erythroblasts, iron is accumulated and stored as $\mathrm{Fe}(\mathrm{III})$ in the earlier stages of erythropoiesis [5].

[1] T van Veen and JA Hunt, Tissue Engin. Regen., Med. 9 (2015), p. 760.

[2] B Vaisman, E Fibach and AM Konijn, Blood 90 (1997) p. 831.

[3] RD Leapman, J. Microsc. 210 (2003) p. 5.

[4] M Fukunaga et al, Proc. Natl. Acad. Sci. USA 107 (2010), p. 3834.

[5] This research was supported by the intramural programs of NIBIB and NIDDK at the NIH.
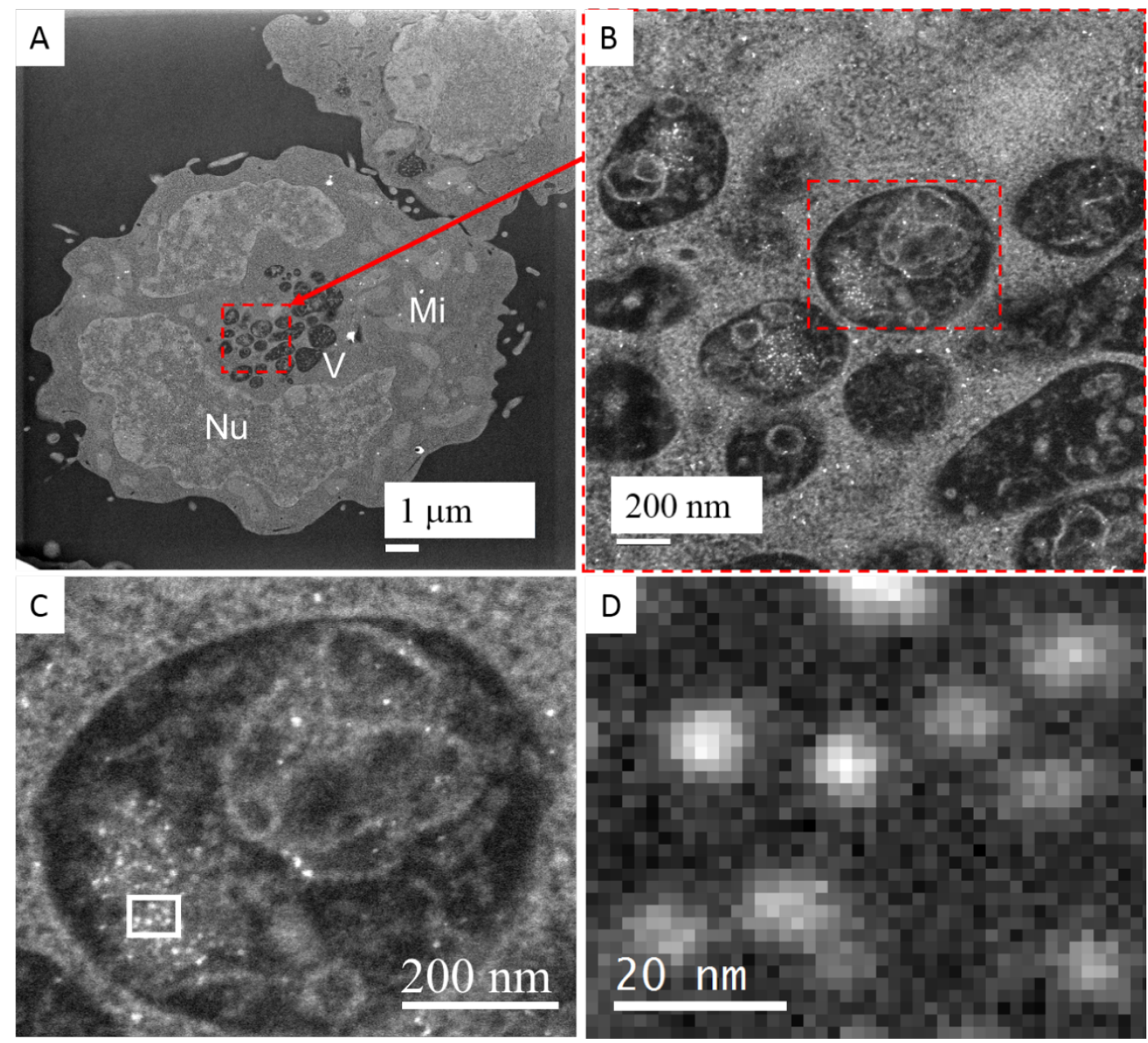

Figure 1. HAADF-STEM and STEM-EELS imaging of $\mathrm{t} 2$ cell during erythroblast differentiation. (A) Overall structure of cell shows nucleus $(\mathrm{Nu})$, mitochondria (Mi) and vesicles (V). (B) and (C) vesicular region of cell at higher magnification shows electron-dense particles of diameters 8 to 10 nm. (D) Calculated Fe distribution obtained by STEM-EELS in sub-region in (C) outlined by white box. 